J. Clin. Chem. Clin. Biochem.

Vol. 16, 1978, pp. 391-395

\title{
Gesamteiweißbestimmung im Serum durch Dichtemessung nach der Biegeschwingermethode
}

\author{
Von H. Holzer, H. Leopold, H. Hinghofer-Szalkay, H. Stübchen-Kirchner und E. Maurer \\ Aus der Medizinischen Universitätsklinik (Vorstand: Prof. Dr. S. Sailer), dem Institut für Physikalische Chemie \\ (Vorstand: Prof. Dr. J. Schurz) und dem Physiologischen Institut (Vorstand: Prof. Dr. Th. Kenner) \\ der Universität Graz
}

(Eingegangen am 28. Dezember 1977/9. März 1978)

Zusammenfassung: Zwischen der Dichte und dem Eiweißgehalt von Serum besteht eine seit langem bekannte, lineare Beziehung. Mit einem neuen Verfahren zur Messung der Dichte, der sogenannten Biegeschwingermethode, gelingt es, diese Beziehung zur Messung der Gesamteiweißkonzentration im Serum zu nützen. Die Methode beruht auf der Bestimmung der Resonanzfrequenz eines mit einer Probe gefüllten, gläsernen, U-förmigen Biegeschwingers. Aus der Verstimmung der Resonanzfrequenz durch die Masse des an der Schwingung teilnehmenden, konstanten Volumens der Probe wird die Dichte bzw. die Eiweißkonzentration berechnet. Trotz der geringen Spezifität der Methode ist der Einfluß von Störsubstanzen wie Glucose, Harnstoff oder Triglyceriden auch bei stark pathologischen Verhältnissen gering. Einfachheit, Schnelligkeit und hohe Genauigkeit in der Dichtebestimmung machen die Biegeschwingermethode zu einem für das Routinelabor empfehlenswerten Verfahren.

\section{Determination of the serum protein concentration based on the measurement of density by the mechanical oscillator technique}

Summary: A linear relation is well established between the density and protein concentration of the serum. The measurement of protein concentration by a new technique for the determination of the absolute density, the mechanical oscillator technique, was investigated.The method is based on the determination of the resonant frequency of a mechanical bending-type, U-shaped glass tube oscillator filled with the sample. The shift in the resonant frequency of the oscillator caused by the mass of the defined volume of the sample taking part in the vibration can be used to calculate the density or protein concentration of the sample. In spite of the low specifity of the method, the interference of substances like glucose, urea or triglycerides is small. Simplicity and rapid results of high accuracy recommend the mechanical oscillator technique for the routine laboratory.

\section{Einfuihrung}

Die verschiedenen Methoḍen zur Beștimmung der Proteinkonzentration in biologischen Flüssigkeiten messen physikalische oder chemische Eigenschaften der Flüssigkeit, die für die Gesamtproteinkonzentration jeweils mehr oder weniger charakteristisch sind. Unspezifische Nachweisverfahren werden von Störsubstanżen wie Lipiden, Kohlehydraten, Harnstoff etc. beeinflußt, proteinspeżifische Methoden ergeben wegen der variablen Zusammensetzung des Gesâmtproteins Ungenauigkeiten. Die neben den chemischen Bestimmungsverfahren (Kjeldahl-, Biuretmethode) zur Verfügung stehenden physikälischen Meßverfahren haben bisher ihre größere methodische Einfachheit mit geringerer Spezifität erkauft. Dies gilt vor allem für die refraktometrische Gesamteiweißbestimmung. Auch die 1930 von Philips und van Slyke $(1,2)$ eingeführte sogenannte Kupfersulfatmethode nach dem Prịnzip des „fallenden Tropfens“ hat wegen der Umständlichkeit der indirekten Dichtebestimmung keinen Eingang in den klinischen Routinebetrieb gefunden, obwohl die Methode auch bei Vorhandensein von Störeinflüssen genaue Resultate erzielt. Die Bestimmung des Serumproteingehaltes nach der genannten Methode beruht auf der indirekten Bestimmung der Dichte von Serum durch Vergleich mit den Dichten einer Reihe von $\mathrm{CuSO}_{4}$-Lösungen gestaffelter Konzentration.

In den letzten Jahren wurde in der Arbeitsgruppe des einen der Autoren (L.) ein neues Verfahren zur Mes- 
sung des Absolutwertes der Dichte (Raumdichte der Masse bzw. Masse der Volumeneinheit) von Flüssigkeiten und Gasen erarbeitet $(3,4)$. Diese sogenannte Biegeschwingermethode wird heute wegen ihrer hohen Präzision für Konzentrationsmessungen reiner, chemisch definierter Flüssigkeiten oder Gase, aber auch zur Feststellung der Zusammensetzung von Meerwasser, Benzin, Ölen, Milch oder Zuckerlösungen angewandt. Wie Leopold et al. gezeigt haben (5), ermöglicht die Biegeschwingermethode heute eine einfache, rasche und genaue Bestimmung der Dichte von biologischen Flüssigkeiten, so daß sich die Methode auch zur Serumproteinbestimmung anbietet (6). Da das Verfahren eine Absolutgenauigkeit der Dichtemessung von besser als $0,01 \mathrm{~g} / 1$ erlaubt und in Anbetracht der hohen Präzision der Methode nach van Slyke (7) haben wir die Eignung der neuen Dichtemeßmethode zur Bestimmung der Gesamtproteinkonzentration im Serum vergleichend untersucht.

\section{Material und Methoden}

\section{Gerät}

Zur Messung der Dichte nach der Biegeschwingermethode wird ein gläserner, U-förmiger Biegeschwinger (Abb. 1) mit der Proben flüssigkeit gefüllt. $\mathrm{Da}$ durch die Geometrie des Schwingers das an der Schwingung teilnehmende Probenvolumen exakt bestimmt ist, besteht - sofern der Schwinger vollständig gefüllt wird keine Notwendigkeit, das Probenvolumen genau zu bemessen. Man bedenke, daß bei der Kalibrierung (s. u.) dasselbe Probenvolumen an der Schwingung teilnimmt; somit entfällt die sonst bei der Dichtemessung notwendige Volumenbestimmung. Die Verstimmung der Resonanzfrequenz des Schwingers kann als direktes Maß für die Masse der Probe genommen werden, so daß daraus Dichte bzw. Eiweißkonzentration berechnet werden können.

Oberflächenspannung, Viskosität und Flïchtigkeit der Probe zeigen keinen Einfluß auf die Genauigkeit der Messung (3). Die heute verfügbaren Dichtemeßgeräte nach der Biegeschwingermethode enthalten einen thermostatisierten Glasschwinger, dessen U-förmiger Hohlzylinder mit der zu messenden Flüssigkeit aus einer Injektionsspritze luftblasenfrei gefullt wird, eine elektronische Einrichtung zur Schwingungsanregung, eine Quarzuhr zur Messung der Schwingungsdauer bzw. Resonanzfrequenz
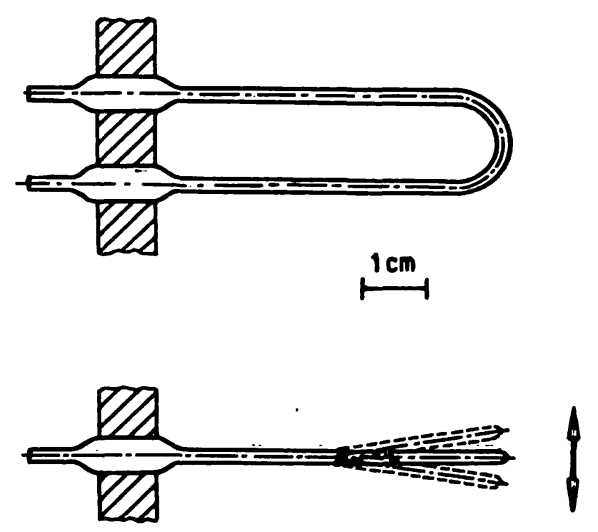

Abb. 1. Schema des Biegeschwingers. und einen Mikrocomputer, der daraus die Dichte oder den Proteingehalt der Probe errechnet und digital anzeigt. Die unten angefuihrten Messungen wurden mit dem Dichtemeßgerät DMA 46 (Hersteller: A. Paar K.G., Graz, Österreich) durchgeführt. Dieses Gerät ist durch einen eingebauten Peltier-Effektthermostaten von einer äußeren Thermostatisierung unabhängig und gewährleistet eine Genauigkeit in der Dichte von $\pm 0,1 \mathrm{~g} / 1$. Eine Dichtemessung dauert 1,7 Sekunden, zuvor ist allerdings für den Temperaturangleich der Probe von Raumtemperatur an die Thermostattemperatur von $20,0^{\circ} \mathrm{C}$ eine Zeitspanne von $20-30$ Sekunden erforderlich. Sämtliche unten angefuịhrten Messungen wurden bei einer Präparattemperatur von $20^{\circ} \mathrm{C}$ durchgefuihrt. Die Kalibrierung des Gerätes erfolgt mit Luft und destilliertem Wasser.

Bestimmungṣmaterial ünd Reagenżien

An 32 normalen Seren und 122 pathologischen Seren wurde die Dichte mit dem angegebenen Meßgerät und die Gesamtproteinkonzentration nach der in den Standard-Methods angegebenen Biuretmethode nach Reinhold (7) bestimmt (Reagenziensatz Merck). Weiter wurde zur Ermittlung der Linearität der Abhängigkeit der Ėiweißkonzentration von der Serumdichte eine wäßrige Verdünnungsreihe einer Albuminlösung hergestellt. Um den Einfluß von Störsubstanzen auf den Zusammenhang zwischen Serumdichte und Proteinkonzentration zu ermitteln, wurde die Dichte von Glucose-, Harnstoff- und Triglyceridlösungen bei $20^{\circ} \mathrm{C}$ als Funktion der Konzentration gemessen. Als Lösungsmittel wurden 3 wäßrige Albuminlösungen auf einen Proteingehalt von 40,70 und $100 \mathrm{~g} / 1$ gebracht und bei variablen Mengen an Glucose, Harnstoff oder Triglyceriden die Konzentration dieser Substanzen, sowie die Dichte der Lösungen gemessen. Harnstoff wurde nach der Berthelot-Reaktion (8) (Reagenziensatz Merck No. 3334), Glucose enzymatisch (9) (Glucosedehydrogenase-Methode, Reagenziensatz Merck No. 14055) und die Triglyceride vollenzymatisch (10) (Test-Kombination Boehringer Mannheim No. 126039) bestimmt.

\section{Ergebnisse}

\section{Linearität}

Sowohl bei den normalen wie bei den pathologischen Seren wurde ein linearer Żusammenhang zwischen der Dichte $\rho(\mathrm{g} / \mathrm{l})$ und der Proteinkonzentration $\mathrm{c}_{\mathrm{P}}(\mathrm{g} / \mathrm{l})$ gefunden, wie Abbildung 2 und 3 zeigen. Die Messungen der Normalseren führen zu folgender Regressionsgeraden:

$$
\rho=1005,2+0,279 c_{\dot{P}}
$$

Daraus läßt sich die Proteinkonzentration als Funktion der Serumdichte errechnen:

$c_{P}=3,58 \rho-3603$

Einer gegebenen Dichteänderung $\Delta \rho$ entspricht daher eine Konzentrationsänderung:

$$
\Delta c_{P}=3,58 \Delta \rho
$$

Diese Beziehung wurde zusätzlich an einer wäßrigen Verdünnungsreihe eines Proteinkonżentrates im Bereich zwischen 1 und $150 \mathrm{~g} / 1$ überprüft. Gleichung 3 sagt aus, daß ein Dichtemeßgerät, welches die Dichte auf $0,1 \mathrm{~g} / 1$ $\left(10^{-4} \mathrm{~g} / \mathrm{cm}^{3}\right)$ genau mißt, die Eiweißkonzentration des Serums auf etwa $0,4 \mathrm{~g} / 1$ genau angeben kann. 


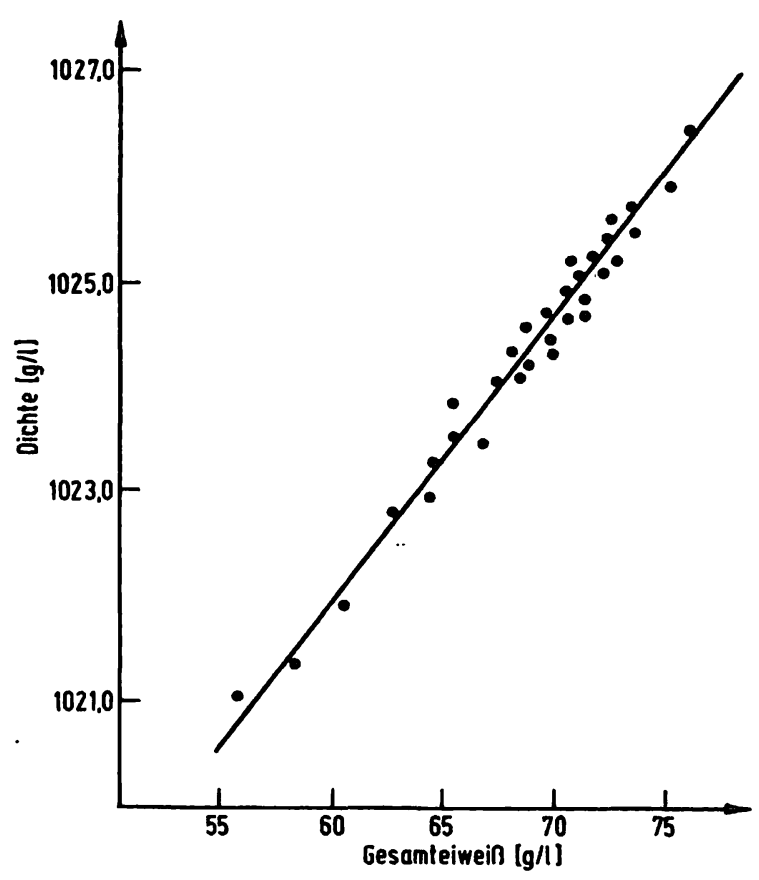

Abb. 2. Beziehung zwischen der Dichte und der nach der Biuretmethode bestimmten Proteinkonzentration.

Normale Seren: $\rho=1005,2+0,279 c_{P}$

$$
\mathrm{r}=0,985, \mathrm{n}=32
$$

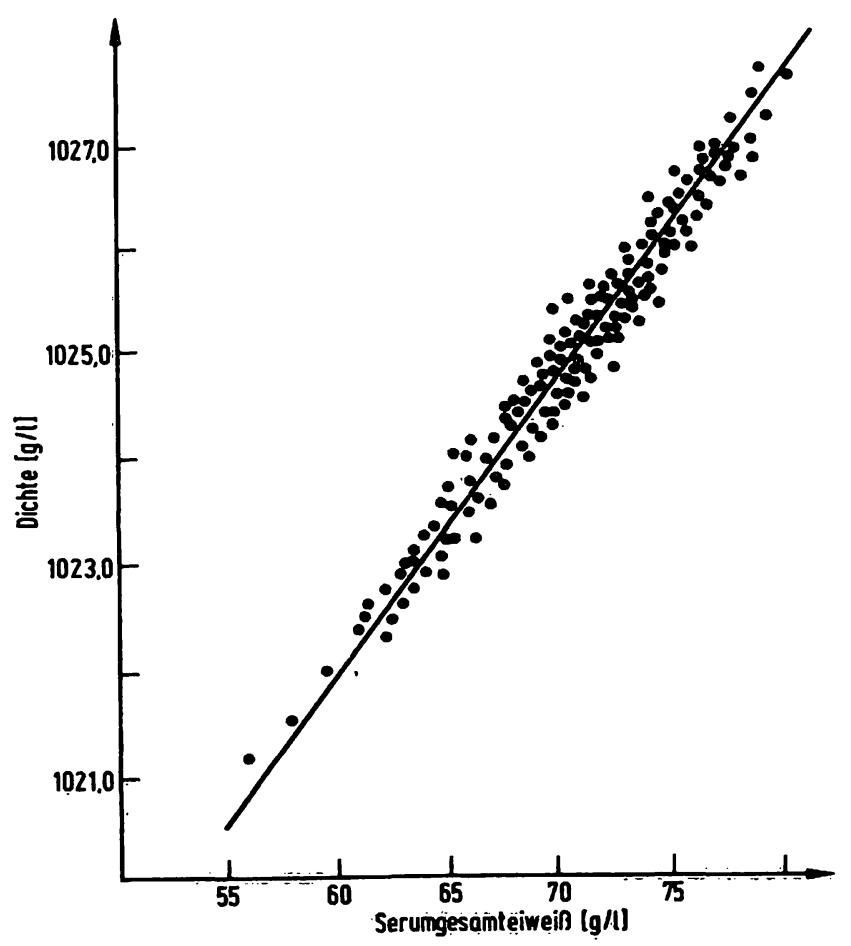

Abb. 3. Beziehung żwischen der Dichte und der nach der Biuretmethode bestimmten Proteinkonzentration.

Pathologische Seren: $\rho=1004,5+0,289 c_{P}$

$$
\mathrm{r}=0,982, \mathrm{n}=122
$$

\section{Richtigkeit}

Die Richtigkeit der Gesamtproteinbestimmung nach der Dichtemethode wurde mit den Kontrollseren Precinorm S, Precipath S (Boehringer Mannheim, Charge 612 und
503) und Validate -A (Labordiagnostika Gödecke, Charge 4 B 502) überprüft. Dabei fand sich auch bei den pathologischen Seren eine Ubereinstimmung zwischen den deklarierten und den nach der Dichtemethode gefundenen Gesamtproteinkonzentrationen bis auf $0,1 \mathrm{~g} / 1$.

\section{Präzision}

Die Präzision wurde an gepoolten Seren in der Serie $(\mathrm{N}=12)$ und von Tag zu Tag $(\mathrm{N}=20)$ überprüft. Bei den Untersuchungen von Tag zu Tag wurden die bei $-20^{\circ} \mathrm{C}$ eingefrorenen Seren frisch aufgetaut. Die Streubreite in der Dichte lag bei $s= \pm 0,31 \mathrm{~g} / 1$, wodurch ein Gesamteiweißfehler von $\pm 1 \mathrm{~g} / \mathrm{l}(\mathrm{VK}=1,43 \%)$ resultiert. Die Präzision in der Serie ergab eine Streubreite in der Dichte von $\mathrm{s}= \pm 0,1 \mathrm{~g} / \mathrm{l}$.

\section{Störfaktoren}

Abbildung 4a zeigt die Dichten von 3 Serumproben der Proteinkonzentration 40,70 und $100 \mathrm{~g} / \mathrm{l}$ als Funktion der variablen Glucosekonzentration $\mathrm{c}_{\mathrm{G}}(\mathrm{mmol} / \mathrm{l})$. Es ergibt sich für

$$
\begin{aligned}
& c_{P}=40 \mathrm{~g} / 1 \\
& \quad \rho=1016,6+0,0694 \mathrm{c}_{\mathrm{G}}, \\
& \mathrm{c}_{\mathrm{P}}=70 \mathrm{~g} / 1 \\
& \quad \rho=1024,9+0,0506 \mathrm{c}_{\mathrm{G}} \text { und für } \\
& \mathrm{c}_{\mathrm{P}}=100 \mathrm{~g} / 1 \\
& \quad \rho=1033,1+0,0320 \mathrm{c}_{\mathrm{G}} .
\end{aligned}
$$

Die Steigung dieser Geraden $\Delta \rho / \Delta c_{G}$ läßt sich als Funktion der Proteinkonzentration im Serum $c_{P}$ ausdrücken:

$$
\frac{\Delta \rho}{\Delta c_{G}}=0,0942-0,00062 c_{P}
$$

Damit läßt sich der Fehler in der Proteinbestimmung, verursacht durch einen bestimmten Glucoseanteil im Serum, abschätzen. Man sieht, daß dieser Fehler mit größer werdender Eiweißkonzentration abnimmt. Aus Gleichung 3 und 4 ergibt sich:

$$
\Delta c_{P}=3,58\left(0,0942-0,00062 c_{P}\right) \Delta c_{G}
$$

Für $5 \mathrm{mmol} / 1$ Glucose und eine Proteinkonzentration von $70 \mathrm{~g} / \mathrm{l}$ ergibt sich ein korrigierbarer Fehler der Proteinbestimmung von $1 \mathrm{~g} / \mathrm{l}$.

Abbildung $4 \mathrm{~b}$ zeigt die Dichten von 3 Serumproben $\left(c_{p}=40,70\right.$ und $\left.100 \mathrm{~g} / 1\right)$ als Funktion der variablen Harnstoffkonzentration $c_{H}(\mathrm{mmol} / \mathrm{l})$. Es gilt für

$$
\begin{gathered}
\mathrm{c}_{\mathrm{P}}=40 \mathrm{~g} / \mathrm{l} \\
\rho=1016,3+0,0156 \mathrm{c}_{\mathrm{H}}, \\
\mathrm{c}_{\mathrm{P}}=70 \mathrm{~g} / 1 \\
\rho=1024,8+0,0123 \mathrm{c}_{\mathrm{H}} \text { und } \\
\mathrm{c}_{\mathrm{P}}=100 \mathrm{~g} / 1 \\
\rho=1033,2+0,0090 \mathrm{c}_{\mathrm{H}} .
\end{gathered}
$$



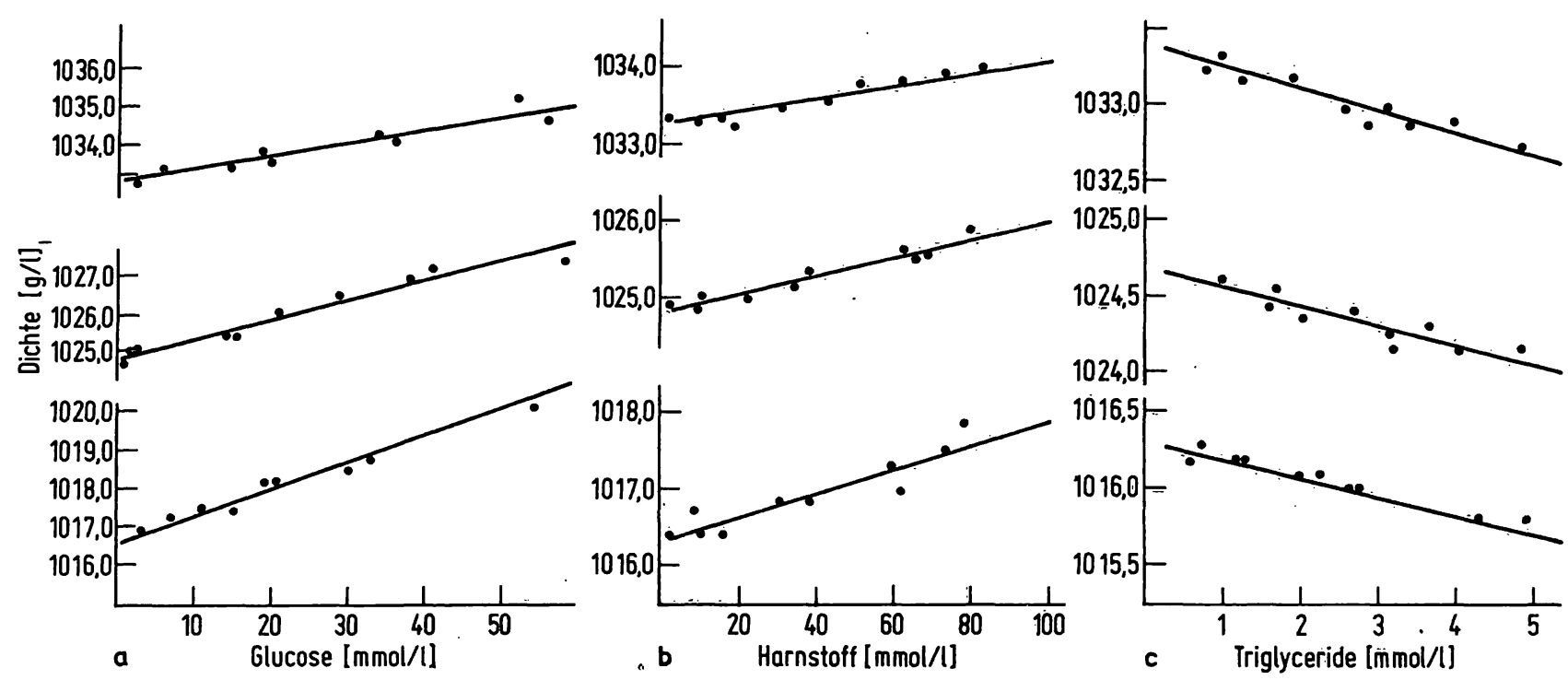

Abb. 4. Beziehung zwischen der Dichte und der Glucose-, Harnstoff- und Triglyceridkonzentration einer Lösung mit 40, 70 und $100 \mathrm{~g} / 1$ Proteingehalt.

a) Glucose, b) Harnstoff, c) Triglyceride.

$$
\begin{aligned}
& \frac{\Delta \rho}{\Delta c_{H}}=0,0200-0,00011 c_{P} \\
& \Delta c_{P}=3,58\left(0,0200-0,00011 c_{P}\right) \Delta c_{H}
\end{aligned}
$$

Für $20 \mathrm{mmol} / 1$ Harnstoff in einem Serum mit $70 \mathrm{~g} / 1$ Proteingehalt ergibt sich ein Fehler in der Proteinbestimmung von $1 \mathrm{~g} / 1$.

Abbildung $4 \mathrm{c}$ zeigt die Dichten von 3 Serumproben als Funktion der variablen Triglyceridkonzentration $c_{T}$. $(\mathrm{mmol} / \mathrm{l})$. Es wurden folgende Beziehungen gefunden:

$$
\begin{aligned}
& \mathrm{c}_{\mathrm{P}}=40 \mathrm{~g} / 1 \\
& \quad \rho=1016,3-0,1123 \mathrm{c}_{\mathrm{T}}, \\
& \mathrm{c}_{\mathrm{P}}=70 \mathrm{~g} / 1 \\
& \quad \rho=1024,7-0,1288 \mathrm{c}_{\mathrm{T}} \text { und } \\
& \mathrm{c}_{\mathrm{P}}=100 \mathrm{~g} / 1 \\
& \quad \rho=1033,4-0,1459 \mathrm{c}_{\mathrm{T}} . \\
& \frac{\Delta \rho}{\Delta \mathrm{c}_{\mathrm{T}}}=-0,0899-0,00056 \mathrm{c}_{\mathrm{P}} \\
& \Delta \mathrm{c}_{\mathrm{P}}=-3,58\left(0,0899+0,00056 \mathrm{c}_{\mathrm{P}}\right) \Delta \mathrm{c}_{\mathrm{T}}
\end{aligned}
$$

Somit bewirken $2,2 \mathrm{mmol} / 1$ Triglyceride in einem Serum mit $70 \mathrm{~g} / 1$ Proteingehalt einen Fehler in der Proteinbestimmung von $-1 \mathrm{~g} / 1$. Man sieht, daß der durch die Triglyceride hervorgerufene Fehler ein umgekehrtes Vorzeichen aufweist als der durch Glucose und Harnstoff bedingte.

\section{Diskussion}

Die vorliegenden Meßergebnisse zeigen, daß mit Hilfe der Biegeschwingermethode die Serumeiweißkonzentration sehr genau gemessen werden kann. Der Einfluß von Störsubstanzen im Serum verursacht einen Meßfehler kleiner als $1 \mathrm{~g} / 1$ bei Normalseren; erst bei extrem pathologischen Verhältnisssen ergibt sịch ein korrigierbarer, größerer Fehler. Treten erhöhte Triglyceridkonzentrationen im Verein mit erhöhten Glucose- oder Harnstoffkonzentrationen auf, so zeigen die Störeinflüsse die Tendenz, einander zu kompensieren.

Die Präzision der Biegeschwingermethode liegt bezüglich der Messung der Dichte weit über dem für die Eiweißbestimmung erforderlichen Auflösungsvermögen und ist etwa um den Faktor 100 besser als die der üblichen Bestimmung der Dichten (5). Der von Heusser \& Schneider (11) bei der Ǔberprüfung der Analysenkalibrierfunktion der Dichtemeßmethoden gefundene Proportionalitätsfaktor $\left(\Delta c_{p} / \Delta \rho=3,60\right)$ liegt bereits niedriger als der von van Slyke angegebene $(3,69)$. Der aus unseren Messungen errechnete Proportionalitätsfaktor $(3,58)$ stimmt mit dem von Doerr (7) theoretisch geforderten Faktor überein. Das bedeutet, daß zwischen den nach der Kjeldahl-Methode und den nach der Dichtemethode bestimmten Eiweißkonzentrationen kein Unterschied besteht.

Die Problematik der Proteinbestimmung mit Hilfe der Dichte liegt nicht in der Präzision, sondern vielmehr in der gegenüber den chemischen Verfahren gèringeren Spezifität. Inwieweit der Unterschied zwischen den normalen und pathologischen Seren durch einen laboreigenen systematischen Fehler der Analysen der Vergleichsmethode oder durch einen systematischen Unterschied zwischen der Vergleichsmethode und der Bezugsmethode bedingt sind, läßt sich aus unserem Versuchsmodell nicht feststellen. Inwieweit Plasmaexpander einen Einfluß auf die Dichtewerte ausüben, ist derzeit Gegenstand weiterer Untersuchungen. Röntgenkontrastmittel haben trotz 
ihrer hohen Dichte (Angiografin 65\%: $\rho=1334,2 \mathrm{~g} / \mathrm{l}$ ) infolge ihrer kurzen Verweilzeit im Blut von nur wenigen Minuten keine Bedeutung als Störfaktor für die Eiweißbestimmung. 30 bis 60 Minuten nach Kontrastmittelinjektion sind die Serumdichtewerte wieder in die Ausgangslage zurückgekehrt (6).

Ein wesentlicher Vorteil der Dichtemethode ist die streng lineare Beziehung auch bei sehr niedrigen wie hohen Meßwerten, der das Verfahren auch zur Proteinbestimmung in Ascites, Pleurapunktat und Liquor cerebrospinalis geeignet erscheinen läßt, worauf eigene Vorversuche hinweisen. Einfachheit und Schnelligkeit des Meßvorgangs sind wesentliche Vorteile gegenüber den chemischen Eiweißbestimmungsmethoden und ermöglichen auch eine problemlose Ausführung der Messung durch ungeschultes Personal im Routinelabor. Die im Routinebetrieb häufigen Pipettier-, Kontaminationsund Ablesefehler sind bei diesem Verfahren ausgeschlossen, da zur Messung ohne genaue Volumenbestimmung lediglich mehr als $0,7 \mathrm{ml}$ Probenvolumen in das Gerät eingebracht werden müssen. Ablesefehler werden durch die direkte digitale Anzeige des Meßwertes weitgehend vermieden, Verunreinigungen des Gerätes werden durch die nach jeder Messung erfolgende automatische Kalibrierung des Gerätes gegen Luft sofort erkannt.

\section{Literatur}

1. Philips, R. A., van Slyke, D. D., Hamilton, P. B., Dole, V. P., Emerson, K. \& Archibald, J. (1950), J. Biol. Chem. 183, $305-309$.

2. van Slyke, D. D., Miller, A., Philipps, R. A., Hamilton, P. B., Dole, V. P., Archibald, R. M. \& Eder, H. A. (1950), J. Biol. Chem. 183, 331-334.

3. Kratky, O., Leopold, H. \& Stabinger, H. (1969), Z. Angew. Phys. 27, 273-277.

4. Leopold, H. (1970), Elektronik 19, 297-302.

5. Leopold, H., Jelinek, R. \& Tilz, G. P. (1977), Biomed. Techn. 10, 231-235.

6. Tilz, G. P. \& Leopold, H. (1972), Wien. Klin. Wochenschr. 84, 697-699.

7. Doerr, P. \& Stamm, D. (1968), diese Z. 6, 304-309.

8. Reinhold, J. G. (1953), Stand. Methods Clin. Chem. 1, 88-97.

9. Fawcett, J. K. \& Scott, J. E. (1969), J. Clin. Pathol. 13, $156-160$.

10. Banauch, D., Brümmer W., Ebeling, W., Metz, H., Rindfrey, H., Lang, H., Leybold, K. \& Rick, W. (1975), diese Z. 13, 101-107.

11. Wahlefeld, A. W. (1974), Methoden der enzymatischen Analyse (Bergmeyer, H. U., ed.), 3. Aufl. Bd. II, 1878-1892, Verlag Chemie, Weinheim/Bergstr.

12. Heußer, H. \& Schneider, J. (1974), Schweiz. Med. Wochenschr. 51, 1327-1330.

Dr. H. Holzer Med. Univ. Klinik Graz Auenbruggerplatz 15 A-8036 Graz 


$$
\cdots
$$$$
\text { , }
$$

$\cdot$

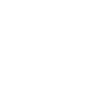

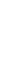

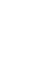

(1)

(

,

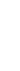

-

